\title{
Hugging Robot for Analyzing Human Behaviour
}

\author{
${ }^{1}$ Mrunali R. Mhaske, ${ }^{2}$ Ankita A. Kudale, ${ }^{3}$ Ruchita Y. Nerkar, ${ }^{4}$ Arpita S. Naik, ${ }^{5}$ Mahendra B. Salunke \\ Department of Computer Engineering, Pimpri Chinchwad College of Engineering \& Research, \\ Ravet, Pune-412101 \\ Email:mrunalimhaske11@gmail.com, kudaleankita98@gmail.com,ruchitanerkar20@gmail.com, \\ arpita.naiksb@gmail.com,mahendra.salunke@pccoer.in
}

\begin{abstract}
Nowadays, various humanoid robots are designed for various applications like manufacturing industries, medical fields, in defense areas where human cannot reach. Robots are used for automating work so as to reduce the manpower. Existing system is, the robot can hug human and the different modules in which we can measure temperature and blood pressure of human. This paper presents Hugging Humanoid for Analyzing Human Behaviour with the help of body temperature and blood pressure. Output of the system will be human will hug the robot and robot will hug back the human and will measure his body temperature and blood pressure and using those parameters it will analyze the human behaviour.
\end{abstract}

Keywords- Arduino Uno, Heart Beat Pulse Sensor, Wifi Module, LM35 Temperature Sensor, Android, Servo Motors, IDE(Integrated Development Environment), Webcam, OpenCV.

\section{INTRODUCTION}

Research on Hugging Humanoid Robot has been currently active topic going on. Because of such research we were motivated to design the Hugging Humanoid which will analyze human behaviour. Hugs makes us feel comfortable, safe and loved. In some situations such as where humans are not available this robot will hug human and help him to reduce the stress. We are going to design a Humanoid Robot. Human will hug the robot. After hugging, the robot will hug again back to human. When robot will hug the human then it will measure body temperature of the human using sensor such as LM35 and heart beat rate using heart beat pulse sensor. These results will be sent to the database using Wifi module and that data will be processed using android application and human behaviour will be analyzed. These data again with the help of application server will be sent to the respective doctor which will help doctor to detect the disorder or behaviour of the human. The following components will be required to design a system.

In this project we have used some Sensors, LED's except of shape, colour or different surfaces. They are also used to measure an approaching or receding object. By using LED's and Buzzers, human easily get to know what action he has to take next time. They are easy to use and gives results fast.

\section{EXISTING SYSTEM}

In the existing system, robot will hug the human if another person is not available to hug. Hug makes us feel warm and comfortable. Robot is actively huggable. It has one jacket, which gives us warm feeling and satisfaction of hugging. It hugs us without any accident [1]. For analyzing human behavior we have referred papers in which, with the help of Arduino uno and using some Arduino devices they are monitoring temperature of the human body and heart beat rate of same human [2] and by monitoring human behavior, it will analyze the human intention [3]. For analyzing human behavior we have to take facial expressions of human, using those expressions human behaviour is analyzed whether human is happy, sad, calm or yawning. For doing non- accidental hug we have to control human robot arm also [6].

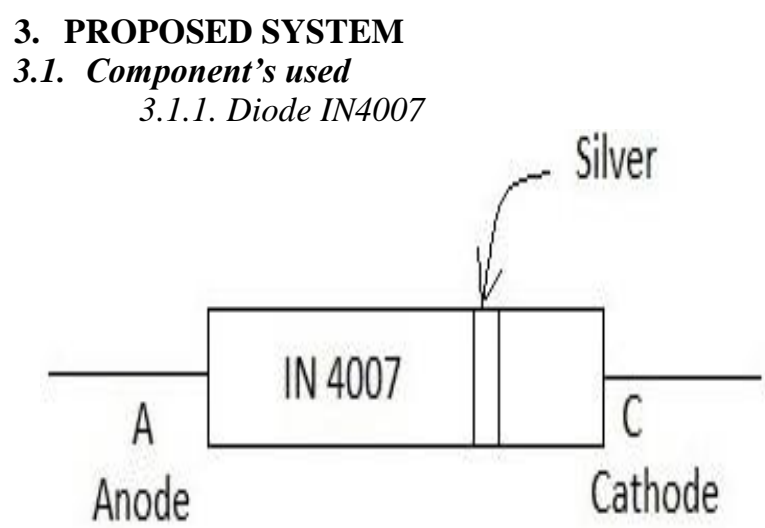

Fig.1 : Diode IN4007

Diode allows current flow from anode to cathode. In diode, current flows in one direction. It's maximum

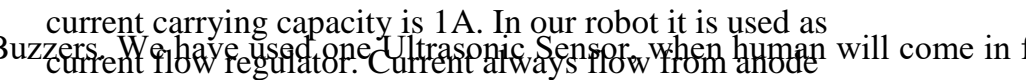
and exit from cathode.

\subsubsection{IC7805}

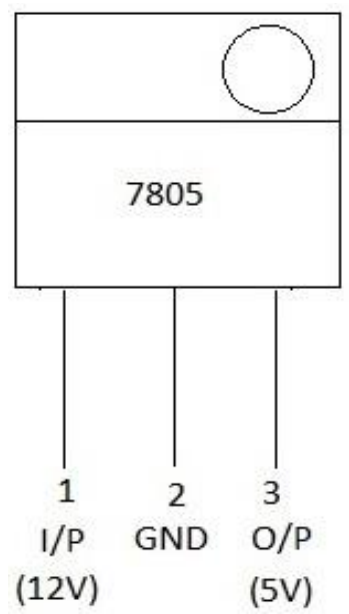

Fig. 2 : IC7805 


\section{International Journal of Research in Advent Technology, Vol.7, No.4, April 2019 \\ E-ISSN: 2321-9637 \\ Available online at www.ijrat.org}

The IC7805 is used for converting $12 \mathrm{~V}$ supply into $5 \mathrm{~V}$ supply. It has three pins. First pin of IC is Input pin which get $12 \mathrm{~V}$ from battery and gives the output of $5 \mathrm{~V}$. Second pin of IC is Ground which is connected to ground. Third pin is Output pin on which we will get the $5 \mathrm{~V}$ supply.

\subsubsection{Ultrasonic Sensor}

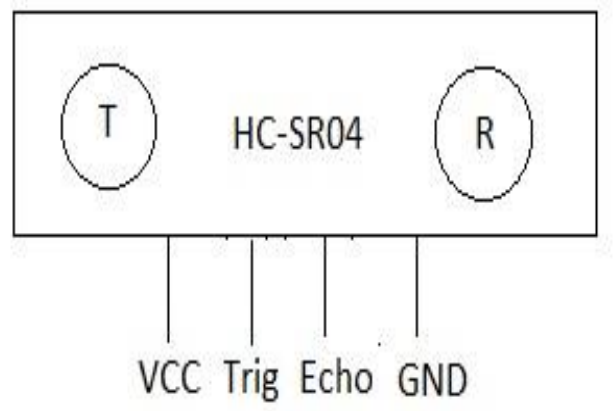

Fig.3 : Ultrasonic Sensor

Ultrasonic sensor is divided into three broad categories: Transmitters, Receiver, Transceivers. Sensors head emits an ultrasonic wave and receive the wave reflected from the target. It measures the distance between the sensor and the human body for hugging movement. Here Trigger pin is input pin and Echo pin is output pin. VCC pin powers the sensor and ground pin is connected to the ground of the system.

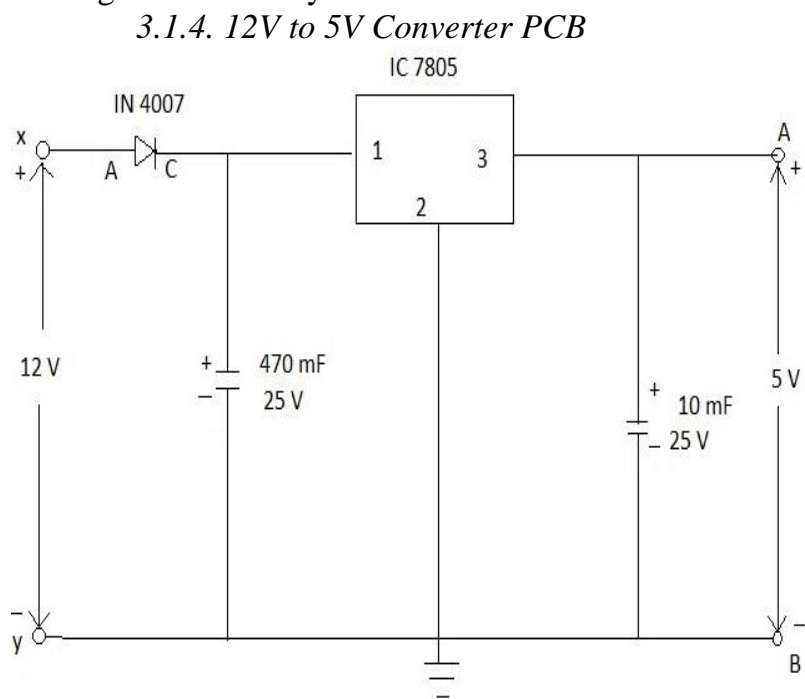

Fig.4 : Circuit of voltage converter

We are giving 12V supply to anode of Diode IN4007. Cathode of diode is shorted in between IC7805 and resister of 470 micro farad. Cathode of diode goes to the input of the IC7805. Ground pin of IC is connected to the ground of the system. Output of the IC is again shorted in between positive supply and resister of 10 micro farad. On that positive supply we will get $5 \mathrm{~V}$.

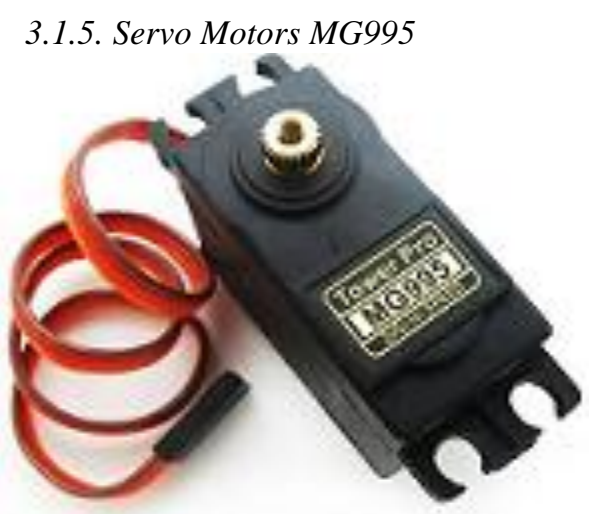

Fig.5 : Servo Motor

Servo Motors will be used for controlling robot's arms for hugging. The rotating shaft of servo motor will control the robot's arm. Robot will move his hand in 90 degrees inwards when it will detect the human and after some delay he will automatic open his hands outwards.

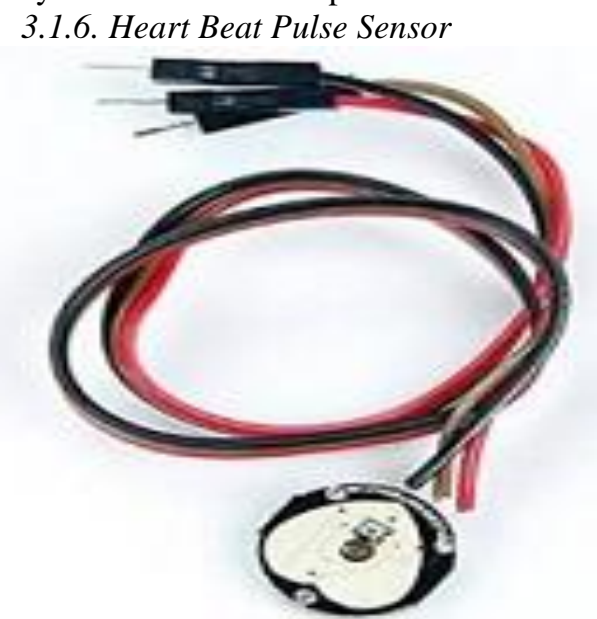

Fig.6 : Heart Beat Sensor

Heart Beat Pulse Sensor which is analog sensor and which is based on photo phlethysmography, will sense the person's heart beat rate and will send it to the Wi-Fi module as output.

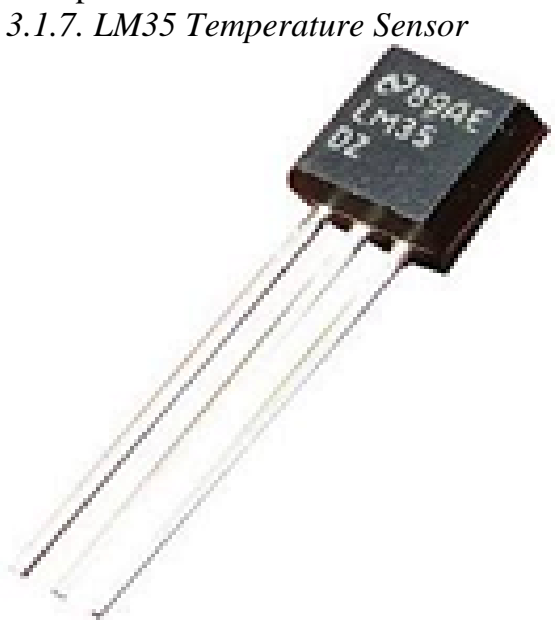

Fig. 7 : LM35 Sensor

LM35 Temperature Sensor which is analog sensor, it consists thermocouples and thermistors that touch body 
to measure the temperature. After sensing the temperature it will send it to Wi-Fi module as output.

\subsection{Architecture Diagram}

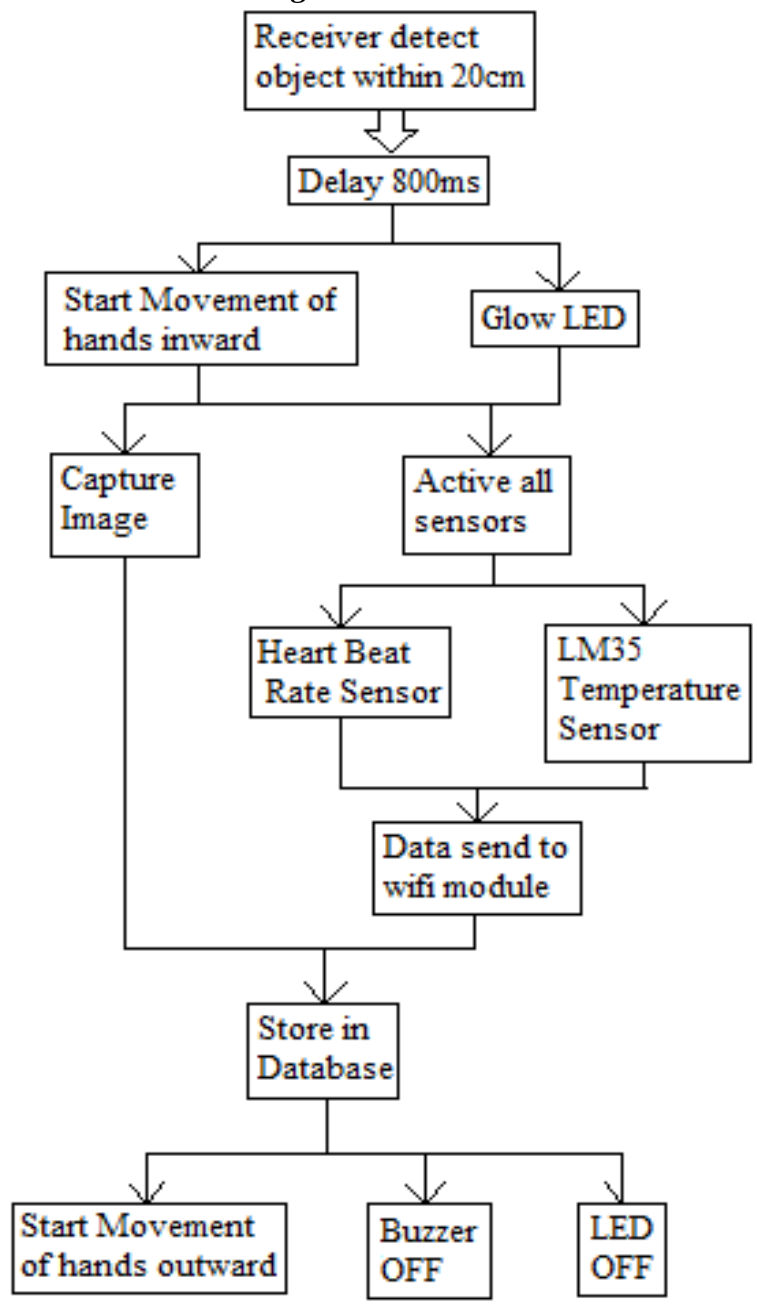

Fig.8 : Architecture Diagram

The above diagram describes about the working of whole module. The transmitter of ultrasonic sensor is continuously transmitting ultrasonic waves, whenever object is detected within $20 \mathrm{~cm}$ range, the receiver receives a signal. As the sensor gets the idea of someone's presence, then it will go for the further process. The further process involves delay time for $800 \mathrm{~ms}$. In this delay process, the human will hug the robot, then LED used will show response by glowing itself and the hands of the robot will start moving in inward direction.

Next process is activation of sensors. The temperature sensor and the pulse rate sensor will get activated and start their functioning. The person whom the robot has hugged, the temperature and pulse rate will be measured of that particular person. The sensors used in this process are LM35 temperature sensor and Heart beat sensor. The data collected by both sensors will be sent to database by using Wi-Fi module and hence the records will be stored in database. The next process is capturing the image. The image of the particular person will be captured and sent to database.And once the whole module has completed its working, then the LED goes off and the buzzer gets activated. This shows that particular person is not detected by ultrasonic sensor now. And once the buzzer starts functioning the hands of robot will start moving in outward direction.

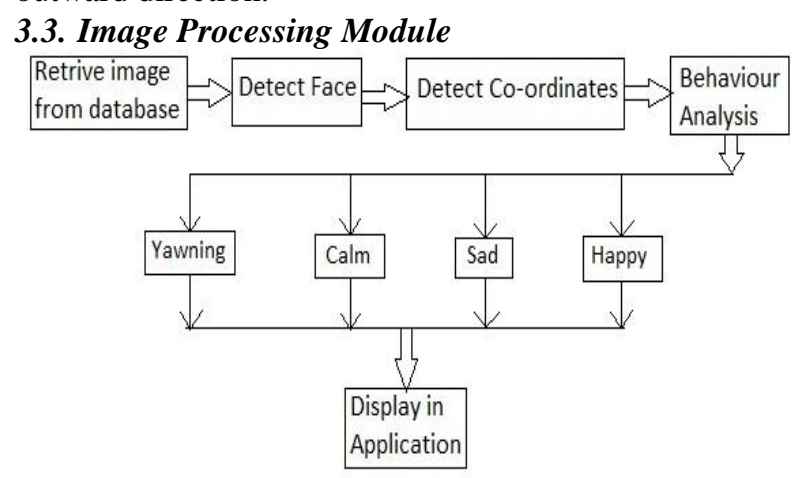

Fig.9 : Image Processing

In image processing, the first step is to capture the image from webcam when the face is detected. By using the algorithm the co-ordinates of face are detected. Based on the facial co-ordinates detected the behaviour of human is analyzed. Then according to the analysis done, the four parameters can be detected. Various facial data of a human gives idea of various emotions. Similarly these images are analyzed. There are four parameters Sad, Happy, Yawning and Calm. The coordinates will be measured and then out of these four parameters which parameter is matching and then what is the behaviour of that particular human will be displayed.

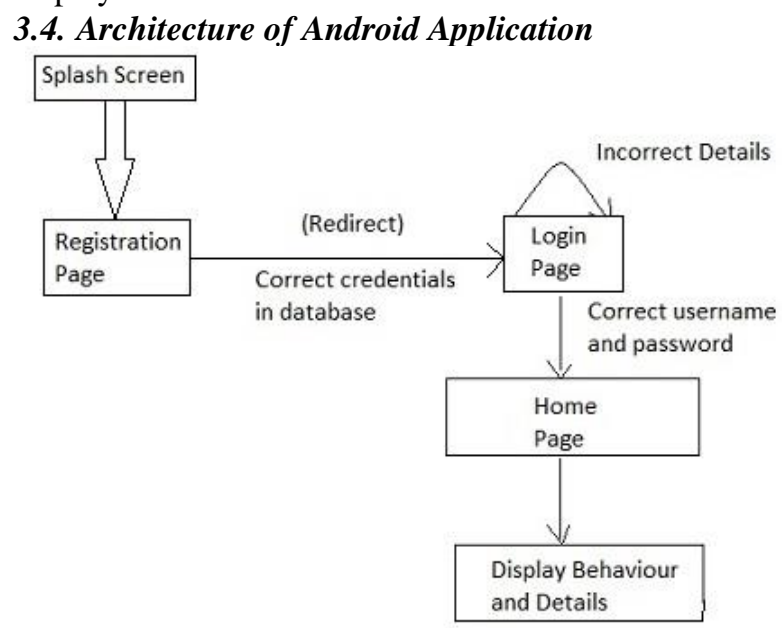

Fig.10 : Architecture of Android Application

The android application starts with the splash screen. Next it goes to registration page where the new user has to register and after that the user will be able to see his details and the further process will be displayed. The registered details are stored in the database and it will redirect to login page.

Then the user has to login first. After login it will redirect to next page where the user will get three options, to see the temperature, or to see the pulse rate or to go towards the result of behaviour analysis. Hence , these parameters measured and saved in the database 
will be fetched in the android application and hence according to the wish of the user, that particular parameter will be displayed.

\section{BEHAVIOUR ANALYSIS}

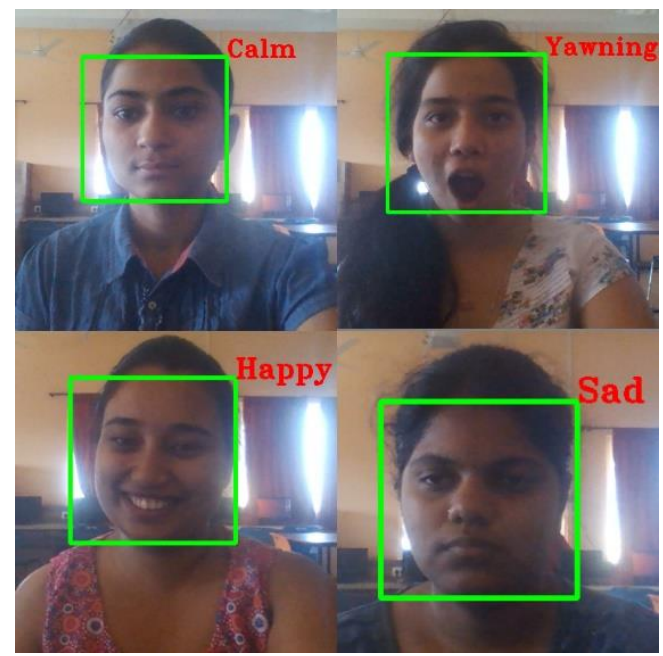

We are using OpenCV for capturing image. After image capturing it will be stored in the database. For analyzing human behaviour we have to retrieve images from database and on the basis of that we are going to analyze human behaviour. As mentioned in fig.9 we have to detect the co-ordinates of the human face and behaviour is analyzed.

There are four types of behaviour which we are going to analysed those are Sad, Happy, Calm, Yawning etc. When whole process is completed then the data i.e. body temperature, heart beat rate and behaviour of human with the help of Wi-Fi module we are going to store in cloud database. After that this whole data is again displayed on android application. User have to login in the module then he will be able to see all parameters in the same module.

\section{REFERENCES}

[1] https://spectrum.ieee.org/automation/robotics/huma noids/the-importance-of-teaching-robots-to-hug

[2] Md. Asaduzzaman Miah, Mir Hussain Kabir, Md. Siddiqur Rahman Tanveer and M. A. H. Akhand; "Continuous Heart Rate and Body Temperature Monitoring System using Arduino UNO and Android Device", Dept. of Computer Science and Engineering Khulna University of Enginnering \& Technology, Proceedings of International Conference on Electrical Information and Communication Technology (EICT 2015).

[3] Tomomasa SAT0 *, Yos hifumi NIS HID A**, Junri IC H I K AWA **, Yotaro HATAMURA** and Hiroshi MIZOGUCHI*,"Active Understanding of Human Intention by a Robot through Monitoring of Human Behavior", *Research Center for Advanced Science and Technology, The Unversity of Tokyo 4-6-1 Komaba, Meguro-ku, Tokyo, 153 Japan.

[4] Subarna Shakya, Suman Sharma1/Abinash Basnet, "Human Behavior Prediction using Facial
Expression Analysis", Department of Electronics and Computer Engineering Central Campus, Institute of Engineering Tribhuvan University Lalitpur, Nepal, International Conference on Computing, Communication and Automation (ICCCA2016).

[5] Tasnim Tarannum*, Anwesha Paul $\dagger$ and Kamrul Hasan Talukdert, "Human Expression Recognition Based on Facial Features", Computer Science and Engineering Discipline Khulna University, Khulna - 9208, Bangladesh, 2016 5th International Conference on Informatics, Electronics and Vision (ICIEV).

[6] Chien-Wei Chen, Rui-Ming Hong, Hung-Yu Wang, "Design of a controlled robotic arm", Department of Electronic Engineering National Kaohsiung University of Applied Sciences Kaohsiung 807, Taiwan, R.O.C, 2016 3rd International Conference on Green Technology and Sustainable Development. 